\section{Prevalência e fatores associados à sintomatologia dolorosa entre profissionais da indústria têxtil}

\section{Prevalence and factors associated with pain symptoms in professionals of the textile industry}

\section{Álvaro Campos Cavalcanti Maciel ${ }^{1}$ \\ Mariana Barros Fernandes ${ }^{1}$ \\ Luciana Souto Medeiros ${ }^{1}$}

'Departamento de Fisioterapia, Centro de Ciências da Saúde, Universidade Federal do Rio Grande do Norte

Endereço para correspondência: Álvaro Campos Cavalcanti Maciel. Rua Moises Gosson, 1442 Lagoa Nova. CEP: 59056-060 Natal-RN. E-mail: alvarohuab@ig.com.br

\section{Resumo}

O trabalho tem um papel importante na vida do homem, pois, além de ser fonte do seu sustento, é onde ele pode sentir-se útil, produtivo e valorizado. Entretanto, quando realizado sob condições impróprias, pode provocar diversos níveis de prejuízo à saúde, como a dor músculo-esquelética, que é caracterizada ainda por possuir origem multifatorial. Desta forma, foi realizado um estudo transversal objetivando analisar a influência dos fatores sociodemográficos e aspectos relacionados ao trabalho e às condições de saúde sobre a sintomatologia dolorosa, em profissionais do setor têxtil. A amostra foi constituída de 162 funcionários que trabalhavam com corte-costura na divisão industrial da ACT (Associação Comunitária para o desenvolvimento do Trairi) no município de Santa Cruz - RN, Brasil. Após análise bivariada e multivariada observou-se associação independente da sintomatologia dolorosa com as variáveis sexo feminino $(\mathrm{p}=0,013)$, tempo de ocupação há mais de seis meses ( $\mathrm{p}=$ $0,005)$, presença de outros problemas de saúde $(\mathrm{p}=0,002)$ e trabalhar em pé $(\mathrm{p}=$ 0,001 ). Nesse contexto, pode-se concluir que houve uma alta prevalência de dor entre os profissionais estudados, e que esta possui associação significativa com vários aspectos relacionados ao trabalho, e alguns fatores sociodemográficos e de saúde, sendo portanto necessária uma atuação interdisciplinar, multiprofissional e intersetorial, de forma a interferir positivamente no processo de trabalho e na saúde do trabalhador.

Palavras-chave: Saúde Ocupacional. Sintomatologia dolorosa. Indústria Têxtil. LER/DORT. Saúde do trabalhador. 


\section{Abstract}

Work has an important meaning in man's life, given it is the most important way for people to support themselves and it is also the only means in which they can feel useful, productive and valuable. However, when work is carried out under inappropriate conditions, it may cause different health injuries, such as muscle-skeletal pain, which is characterized by a multifactorial origin. Thus, a cross-sectional study was carried out aimed at analyzing the influence of sociodemographic factors, work and health conditions on pain symptoms in professionals of the textile industry. The sample comprised 162 sewing machine operators from ACT's (Community Association for the Development of Trairi) industrial division in Santa Cruz - RN, Brazil. After bivariate and multivariate analyses, we found an independent association between pain symptoms and variables such as female gender $(p=0.013)$, more than six months in the same occupation $(\mathrm{p}=0.005)$, other health problems $(\mathrm{p}=$ 0.002 ), and working in a standing position $(\mathrm{p}=0.001)$. The study identified a high prevalence of pain symptoms in textile industry professionals and a significant association with many work-related aspects and some sociodemographic and health factors, making an interdisciplinary, multiprofessional and intersectorial action necessary to promote positive interference in the work process and occupational health.

Keywords: Occupational Health. Pain Symptoms. Textile Industry. Work-Related Musculoskeletal Disease. Worker's health.

\section{Introdução}

O trabalho tem um papel importante na vida do homem, pois, além de ser fonte do seu sustento, é onde este pode se sentir útil, produtivo e valorizado, tendo sua auto-estima elevada, passando a contar com a possibilidade concreta de auto-realização ${ }^{1}$.

Entretanto, quando realizado sob condições inadequadas, o trabalho pode ser nocivo, prejudicando a saúde, provocando doenças, levando à inatividade, encurtando a vida e até causando a morte ${ }^{2}$.

Neste contexto, o impacto socioeconômico dos distúrbios osteomusculares ocupacionais vem crescendo de forma preocupante, visto que, em todo o mundo, a prevalência desta patologia vem atingindo proporções epidêmicas ${ }^{3}$.

Atualmente, diversos estudos vêm sendo realizados com o objetivo de analisar a relação existente entre o desenvolvimento das atividades profissionais e o aparecimento de problemas músculo-esqueléticos, cujos resultados mostram que a execução de algumas tarefas contribuem, de forma mais significativa para o surgimento de tais distúrbios ${ }^{4,5}$.

Como fatores de risco relacionados ao trabalho consideram-se as posturas e os movimentos inadequados, repetições, vibrações, carga estática e dinâmica, intervalo de descanso e os aspectos ambientais (ruído, iluminação, temperatura etc.) como os grandes preditores no surgimento de quadros álgicos ${ }^{6}$.

Em indústrias do setor têxtil observase que a postura adotada no trabalho influi de maneira significativa no surgimento de quadros dolorosos, principalmente na coluna vertebral e nas pernas ${ }^{2}$.

Considerando-se que a eficácia de qualquer medida depende diretamente de sua capacidade para atingir, eliminar ou minimizar os fatores desencadeadores de doenças, é essencial, inicialmente, conhecê-los e analisá-los. Sendo assim, este trabalho objetivou verificar a prevalência e os fatores associados à sintomatologia dolorosa entre os profissionais do setor têxtil. 


\section{Metodologia}

O estudo foi realizado na divisão industrial da Associação Comunitária para o desenvolvimento do Trairí (ACT), no município de Santa Cruz / RN - Brasil, localizado a $120 \mathrm{~km}$ de Natal (capital do Estado), na região Nordeste do país. O ramo de atividade desenvolvido nessa indústria é a confecção, sendo o vestuário geral o principal produto.

De acordo com a problemática do estudo, optou-se por realizar uma pesquisa exploratória com um desenho transversal. A amostra foi constituída pelos 162 profissionais que trabalhavam com corte-costura em uma das etapas específicas da preparação do produto final (preparação da frente, preparação do traseiro, montagem, acabamento e atividades manuais).

Como instrumento de coleta dos dados foi elaborado um questionário multidimensional que constou dos seguintes itens:

- Aspectos sociodemográficos: idade, sexo, estado civil, número de filhos e grau de instrução.

- Condições de trabalho: cargo na empresa, tempo que trabalha na empresa e na ocupação atual, principal postura no trabalho e aquela que cause dor, realização de horas extras, realização de pausas, falta ao trabalho e motivo, salário, satisfação com o salário e com a profissão e realização de trabalho fora da empresa.

- Saúde geral: Índice de massa corporal (IMC), problemas de saúde, tratamentos realizados, exercícios físicos, ocasiões em que sente dores.

A coleta de dados foi realizada nos galpões da divisão industrial, durante a jornada de trabalho dos funcionários e foi antecedida por um estudo piloto, que objetivou a correção de possíveis erros, testagem e calibração dos entrevistadores.

Os dados obtidos foram introduzidos em um banco de dados do programa estatístico SPSS (versão 10.0). A análise foi realizada mediante uma abordagem descritiva e outra analítica. $\mathrm{Na}$ abordagem descritiva foi feita a distribuição de freqüência absoluta e relativa para variáveis categóricas e média, com desvio padrão (DP) para variáveis contínuas. Na abordagem analítica realizou-se uma análise bivariada utilizando o teste do qui-quadrado de Pearson, para se observarem as possíveis associações entre as variáveis independentes com a variável dependente “presença de sintomatologia dolorosa". Em seguida, realizada análise multivariada, por regressão logística binária, utilizando análise hierarquizada. Mediante a estratégia estabelecida de associações entre as dimensões estudadas, que foram as sociodemográficas, condições de trabalho e condições de saúde, foram elaborados três modelos explicativos de regressão logística binária, introduzindo as variáveis em forma de blocos, na ordem acima descrita, permanecendo no modelo subseqüente apenas as variáveis que tiveram significância estatística $(\mathrm{p}<0,05)$ no modelo anterior. O critério de saída para todas as variáveis introduzidas em cada modelo foi $\mathrm{p}<0,10$. Ao final, chegou-se a um Modelo Final de regressão com apenas aquelas variáveis de maior significância estatística. O método de introdução das variáveis nos modelos adotados foi o "backward stepwise”. Considerou-se um nível de significância $\mathrm{p}<0,05$ e intervalo de confiança (IC) de 95\%, com cálculo dos odds ratios ajustados.

A pesquisa foi realizada dentro dos padrões éticos, garantindo o respeito à dignidade humana, segundo o parecer 49/01 emitido pelo Comitê de Ética em Pesquisa local.

\section{Resultados}

Um total de 162 sujeitos foram entrevistados, sendo 53 (32,7\%) do sexo masculino e 109 (67,3\%) do sexo feminino, com idade variando de 16 a 49 anos, e média de $26,12(\mathrm{DP}= \pm 6,85)$, correspondendo a $100 \%$ da população inicial. 
No que diz respeito ao grau de instrução, encontraram-se 3 (1,9\%) pessoas analfabetas ou semi-analfabetas e 159 (98,1\%) alfabetizadas, ou seja, que possuíam no mínimo o ensino fundamental incompleto. Sobre o estado civil, 99 (61,3\%) eram solteiras (foram incluídas nesta categoria as pessoas solteiras, viúvas e divorciadas) e $63(38,9 \%)$ casadas (casadas ou que viviam em união consensual). Quanto ao número de filhos, 71 (43,8\%) não tinham filhos.

A principal atividade laboral identificada foi à confecção de roupas, sendo realizada através de cinco diferentes funções, onde 53 funcionários $(32,7 \%)$ trabalhavam na preparação da frente, 51 (31,5\%) na montagem, $34(21,0 \%)$ na preparação do traseiro, $15(9,3 \%)$ no acabamento e 9 $(5,6 \%)$ em trabalhos manuais.

Analisando-se o tempo em que os funcionários trabalhavam naquelas funções, observou-se que a maioria (58,0\%) estava ali há mais de seis meses.

Durante a jornada de trabalho 113 funcionários $(69,8 \%)$ passavam mais tempo na posição sentada e 49 (30,2\%) em pé.

Em relação às pausas para descanso, 40 $(20,4 \%)$ indivíduos relatavam fazê-las, enquanto 122 (75,3\%) não as faziam. A realização de horas extras era comum a 129 (79,6\%) funcionários, enquanto 33 (20,4\%) não as realizavam. Dos 162 entrevistados, $144(88,9 \%)$ não executavam outro trabalho fora da empresa e 18 (11,1\%) possuíam outra ocupação, entre as quais destacaram-se a criação de animais de corte, comércio varejista, trabalhos domésticos e pintura.

Observou-se que 14 (8,6\%) indivíduos haviam faltado ao emprego nos últimos seis meses por sintomatologia dolorosa, relacionada ao trabalho e $148(91,4 \%)$ não tiveram faltas por esse motivo. As queixas mais comuns foram dores na coluna, pernas e braços, respectivamente.

Em relação à satisfação com o salário, 105 (64,8\%) sujeitos julgavam satisfatória a quantia recebida, enquanto 57 (35,2\%) possuíam opinião contrária. Quanto à sa- tisfação com a profissão exercida, 120 $(74,1 \%)$ funcionários afirmaram estar realizados profissionalmente e 42 (25,9\%) eram contrários a esta opinião.

Sobre as condições de saúde dos entrevistados, $129(79,6 \%)$ estavam com IMC considerado normal e $33(20,4 \%)$ estavam acima do peso. Em relação aos problemas de saúde, 70 (43,2\%) afirmaram possuir alguma patologia ou distúrbio e 92 (56,8\%) negaram essa condição. As patologias mais citadas foram: problemas na coluna, dores nas pernas, problemas nos joelhos e dormência nos braços, respectivamente. Dos sujeitos que afirmaram ter problemas de saúde, 23 (32,8\%) relataram já haver realizado algum tipo de tratamento. No que diz respeito à prática de exercícios físicos, encontraram-se 51 (31,5\%) praticantes regulares dessas atividades.

Quanto à presença de dor, 61 (37,7\%) funcionários relataram ausência de quadros álgicos ou sentiam dores em apenas uma região corporal, enquanto 101 (62,3\%) referiram sintomatologia dolorosa em mais de um local. Em relação às regiões de maiores queixas de dor, as porções cervical e torácica da coluna vertebral, pernas e ombros foram, respectivamente, as mais citadas.

$\mathrm{Na}$ análise entre as variáveis sociodemográficas e a presença de sintomatologia dolorosa, observou-se associação significativa apenas com a variável sexo $(\mathrm{p}<$ 0,001) (Tabela 1).

Sobre as variáveis relacionadas às condições de trabalho, encontrou-se associação significativa para o tempo de ocupação ( $\mathrm{p}<0,001)$, onde $77,9 \%$ dos indivíduos com mais de seis meses de trabalho na empresa referiram dor em mais de uma região corporal. Observou-se também uma maior prevalência de sintomatologia dolorosa em funcionários que trabalhavam em pé $(19,6 \%)$ ( $p=0,003)$, em indivíduos que realizavam horas extras $(67,4 \%)$ $(\mathrm{p}=0,008)$ e naqueles que relatavam já terem faltado ao emprego por sintomatologia dolorosa relacionada ao trabalho $(85,7 \%)(\mathrm{p}=0,05)$ (Tabela 2). 
Com relação às variáveis de condições de saúde, percebeu-se um resultado significativo na prevalência de dor nos sujeitos que afirmavam ter algum problema de saúde $(80,0 \%)(p<0,001)$ e naqueles que não praticavam atividade física $(\mathrm{p}=0,002)$ (Tabela 3).

$\mathrm{Na}$ regressão logística, encontrou-se que o sexo feminino ( $\mathrm{p}=0,013$ ), os funcionários que trabalhavam há mais de seis

Tabela 1 - Análise bivariada entre variáveis sociodemográficas e a presença de dor.

Table 1 - Bivariate analysis of sociodemographic variables and presence of pain

\begin{tabular}{lccccc}
\hline \multicolumn{5}{c}{ PRESENÇA DE DOR } \\
& $\mathrm{n}$ & $\begin{array}{c}\text { Nenhuma / uma região } \\
\%\end{array}$ & $\mathrm{n}$ & $\%$ & $\mathrm{p}$ \\
\hline Sexo & 29 & 26,6 & 80 & 73,4 & 0,000 \\
Feminino & 32 & 60,4 & 21 & 39,6 & \\
Masculino & & & \\
\hline
\end{tabular}

Tabela 2 - Análise bivariada entre variáveis de condições de trabalho e a presença de dor. Table 2 - Bivariate analysis of work condition variables and presence of pain

\begin{tabular}{|c|c|c|c|c|c|}
\hline & \multicolumn{4}{|c|}{ PRESENÇA DE DOR } & \multirow[b]{3}{*}{$\mathrm{p}$} \\
\hline & \multicolumn{2}{|c|}{ Nenhuma / uma região } & \multicolumn{2}{|c|}{ Mais de uma região } & \\
\hline & $\mathrm{N}$ & $\%$ & $\mathrm{n}$ & $\%$ & \\
\hline \multicolumn{6}{|l|}{ Tempo de ocupação } \\
\hline 0 a 6 meses & 46 & 48,9 & 48 & 51,1 & \multirow[t]{2}{*}{0,000} \\
\hline Mais de 6 meses & 15 & 22,1 & 53 & 77,9 & \\
\hline \multicolumn{6}{|l|}{ Posição de trabalho } \\
\hline Sentado & 51 & 45,1 & 62 & 54,9 & \multirow[t]{2}{*}{0,003} \\
\hline Em pé & 10 & 20,4 & 39 & 79,6 & \\
\hline \multicolumn{6}{|l|}{ Horas extras } \\
\hline Sim & 42 & 32,6 & 87 & 67,4 & \multirow[t]{2}{*}{0,008} \\
\hline Não & 19 & 57,6 & 14 & 42,4 & \\
\hline \multicolumn{6}{|c|}{$\begin{array}{l}\text { Falta ao emprego por } \\
\text { sintomatologia dolorosa } \\
\text { relacionada ao trabalho }\end{array}$} \\
\hline Sim & 2 & 14,3 & 12 & 85,7 & \multirow[t]{2}{*}{0,05} \\
\hline Não & 59 & 39,9 & 89 & 60,1 & \\
\hline
\end{tabular}

Tabela 3 - Análise bivariada entre variáveis de condições de saúde e a presença de dor. Table 3 - Bivariate analysis of health status variables and presence of pain

\begin{tabular}{|c|c|c|c|c|c|}
\hline & & PRESEN & $\mathrm{DOR}$ & & \\
\hline & Nenh & região & Mais & egião & \\
\hline & $\mathrm{N}$ & $\%$ & $\mathrm{n}$ & $\%$ & $p$ \\
\hline Problem & & & & & \\
\hline Sim & 14 & 20,0 & 56 & 80,0 & 0,000 \\
\hline Não & 53 & 57,6 & 39 & 42,4 & \\
\hline Exercíci & & & & & \\
\hline Sim & 27 & 54,9 & 24 & 45,1 & 0,002 \\
\hline Não & 34 & 29,7 & 77 & 70,3 & \\
\hline
\end{tabular}


meses na mesma ocupação $(p=0,005)$ e os que possuíam outros problemas de saúde $(p=0,002)$, tiveram cerca de três vezes mais chances de apresentar dor em mais de uma região corporal. Da mesma forma, os indivíduos que trabalhavam em pé $(\mathrm{p}=0,001)$ tiveram cerca de cinco vezes mais chances de se apresentar com mais de uma região corporal com sintomatologia dolorosa (Tabela 4).

\section{Discussão}

O presente trabalho possibilitou constatar a situação de saúde atual, dentro das dimensões estudadas, dos funcionários que trabalham com corte-costura nos galpões da ACT, no município de Santa Cruz - RN, e sua relação com a sintomatologia dolorosa. Isto foi possível pela eleição do tipo de pesquisa, pois os estudos transversais possibilitam uma reflexão sobre a situação encontrada no momento da avaliação, mostrando um retrato instantâneo da amostra estudada.

De acordo com os resultados, pôde-se constatar que a maior parte da amostra era do sexo feminino, com média de idade de 26,12 anos. No coletivo dos operadores de máquinas de costura, as mulheres representam a grande parcela, como pode ser constatado em outros estudos ${ }^{4,8,9}$.

Ao analisar a estrutura etária de trabalhadores portadores de LER/DORT percebe-se uma clara predominância de indiví- duos na faixa de 20 a 39 anos, o que, numericamente, corresponde a 59,9\% dos casos diagnosticados ${ }^{3}$.

O fato de a maioria dos entrevistados ser alfabetizada contribuiu significativamente para o estudo, permitindo uma fácil compreensão do questionário avaliativo aplicado, no que se refere à interpretação dos questionamentos e às instruções dadas pelos pesquisadores.

Em relação à estrutura familiar, a maior parte da amostra era constituída por solteiros e por aqueles que possuíam pelo menos um filho. $\mathrm{O}$ fator filho representa um dos principais causadores de dores relacionadas aos membros superiores e à região cervical em profissionais de costura ${ }^{10}$.

A respeito da análise das condições de trabalho, mais da metade dos funcionários trabalhava nesse ofício há mais de seis meses. Ao serem analisados estudos anteriores, observa-se que, em geral, o tempo de ocupação na mesma função de operador de máquina de costura é superior a um ano $^{10,11}$.

Durante a jornada de trabalho, a maioria dos indivíduos fazia hora extra, não realizava pausas para descanso e passava a maior parte do tempo sentada. A postura sentada provoca uma imobilização das peças do esqueleto em uma atitude de conjunto, resultante de um trabalho muscular estático, principalmente da musculatura do dorso. Essa condição adversa provoca o desenvolvimento de processos infla-

Tabela 4 - Resultado da análise multivariada de regressão logística, Modelo Final para associações independentes com a presença de sintomatologia dolorosa.

Table 4 - Results of logistic regression multivariate analysis, Final Model for independent associations and presence of pain symptoms.

\begin{tabular}{|c|c|c|c|c|}
\hline Variáveis & Referência & $\mathrm{p}$ & $\mathrm{OR}_{\text {ajustada }}$ & IC 95\% \\
\hline \multicolumn{5}{|l|}{ Sexo } \\
\hline Feminino & Masculino & 0,013 & 3,08 & $1,20-7,51$ \\
\hline \multicolumn{5}{|l|}{ Tempo no cargo } \\
\hline Mais de seis meses & Menos de seis meses & 0,005 & 3,18 & $1,4-7,15$ \\
\hline \multicolumn{5}{|l|}{ Posição de trabalho } \\
\hline Em pé & Sentado & 0,001 & 5,05 & $1,9-12,83$ \\
\hline \multicolumn{5}{|l|}{ Problema de saúde } \\
\hline Sim & Não & 0,002 & 3,68 & $1,6-8,48$ \\
\hline
\end{tabular}


matórios nas estruturas osteomusculares com sintomatologia dolorosa associada ${ }^{12}$. Há muitos anos preconiza-se a existência de períodos de pausa para repouso, a fim de garantir a recuperação física de um processo de fadiga muscular orgânica e microtraumas de estruturas como tendões, bainhas e bolsas sinoviais ${ }^{8}$.

As faltas ao emprego por sintomatologia dolorosa relacionada ao trabalho são uma realidade para muitos funcionários da ACT. O absenteísmo causado por distúrbios músculo-esqueléticos é uma constante no meio industrial e relacionase com vários fatores, como o avanço e a estrutura da indústria e o desenvolvimento socioeconômico da região, tendo aumentado consideravelmente nos últimos cinco anos ${ }^{13,14}$.

Dos entrevistados, grande parte estava satisfeita com o salário recebido e com a profissão exercida. Os aspectos psicosociais interferem nos quadros de LER/ DORT, tendo como sintomas comuns: falta de reconhecimento do trabalho realizado, perda de identidade, estado de estresse, esgotamento acentuado e as próprias limitações impostas pela doença ${ }^{15}$. Atualmente, sabe-se que a insatisfação com o trabalho é um fator associado à presença de dores na região cervical e ombros pela tensão muscular gerada ${ }^{16,17}$.

Quanto às condições de saúde, os funcionários, em sua maioria, apresentaram IMC normal, dado que concorda com o estudo de Moraes et al. (2002). Em relação aos problemas gerais de saúde, a maior parte dos indivíduos afirmava possuir alguma patologia e, destes, apenas $32,8 \%$ já haviam realizado algum tipo de tratamento. Em outro estudo, verificou-se que $44,0 \%$ dos trabalhadores precisavam de intervenção terapêutica, incluindo tratamento medicamentoso e fisioterapêutico ${ }^{8}$.

Observou-se uma alta prevalência de dor entre os profissionais analisados, sendo a coluna vertebral (níveis cervical e torácico), pernas e ombros, respectivamente, os locais mais citados. Muitos estudos epidemiológicos sobre distúrbios músculo-esqueléticos agudos e crônicos em costureiras mostraram que as regiões do corpo com maiores queixas são a coluna cervical e os ombros ${ }^{11,17,18}$. Em trabalhadores da indústria em geral, a queixa de dor nas pernas por distúrbios músculo-esqueléticos é freqüente, sendo referida por mais da metade dos indivíduos ${ }^{19}$.

Uma vez exposto e analisado o perfil da amostra estudada, faz-se necessária uma discussão aprofundada em relação à análise bivariada e multivariada. Sobre as variáveis relacionadas às condições de trabalho, verificou-se que os funcionários que realizavam horas extras e os que relatavam já terem faltado ao emprego por sintomatologia dolorosa relacionada ao trabalho apresentaram mais dor, quando comparados àqueles em situação oposta, dados condizentes com um estudo anterior ${ }^{11}$.

Os indivíduos que trabalhavam há mais de seis meses na mesma ocupação apresentaram cerca de três vezes mais chances de desenvolver sintomatologia dolorosa em mais de uma região corporal. De fato, a prevalência de dores persistentes nos ombros e coluna cervical aumenta com o decorrer do tempo de emprego em operadores de máquinas de costura. A ocorrência de LER/DORT entre os trabalhadores com até dois anos de ocupação permanece elevada desde 1996, representando $20 \%$ dos casos diagnosticados ${ }^{3}$.

Outro fator significativo foi a prática regular de exercícios físicos, visto que a maioria dos sedentários apresentou queixas de dor, enquanto menos da metade dos funcionários praticantes de atividades físicas relataram essa sintomatologia. A atividade física causa adaptações circulatórias e metabólicas, resultando em alterações benéficas na musculatura esquelética e tecidos conectivos. Essas alterações contribuem para a diminuição do risco de surgimento de incapacidades e injúrias osteomusculares ${ }^{20}$.

A associação significativa identificada entre o sexo feminino e a sintomatologia dolorosa é condizente com o resultado obtido em outros estudos ${ }^{3,21}$, e pode ser 
explicada pelo fato de que os distúrbios do sistema músculo-esquelético ocorrem, freqüentemente, quando a demanda física do trabalho excede a capacidade física do trabalhador. Sob esse aspecto, a diferença de massa muscular, composição corporal e tamanho das mulheres em relação aos homens pode representar, para esse grupo, um fator de risco predisponente da sintomatologia dolorosa ${ }^{8,9}$.

Os indivíduos cujo trabalho era realizado, principalmente na postura em pé, apresentaram cinco vezes mais chances de possuir dor em mais de um local. De fato, as atividades desenvolvidas por esses profissionais exigem um baixo nível de força muscular e permitem a adoção de posturas desalinhadas ou estáticas por um período prolongado de tempo, representando riscos biomecânicos e apresentando uma forte relação com os distúrbios osteomusculares relacionados ao trabalho ${ }^{11}$. Trabalhos realizados, predominantemente na postura em pé, principalmente quando associados à rotação e inclinação do tronco para frente, são considerados fatores de risco de dor no pescoço, ombros e pernas ${ }^{22,23}$.

Com relação às condições de saúde, a sintomatologia dolorosa apresentou associação significativa com a alta prevalência de indivíduos que afirmaram possuir algum problema de saúde. A presença de transtornos patológicos torna o indivíduo mais propenso a sentir dor. Dessa forma, os principais fatores de riscos individuais predisponentes aos quadros álgicos são as experiências patológicas anteriores e sintomas similares em outras partes do corpo ${ }^{17}$.
As demais variáveis, como a satisfação com o salário ou com a profissão, a realização de outros trabalhos fora da empresa, o índice de massa corporal, a realização de pausas para descanso, o cargo na empresa, o número de filhos, o estado civil e o grau de instrução, não apresentaram resultados estatisticamente significativos. No entanto, a literatura enquadra essas variáveis como fatores de risco para a presença de dor relacionada ao trabalho $8,11,16$.

Diante do apresentado, a partir da metodologia empregada e dos resultados obtidos, os objetivos propostos foram alcançados e os aspectos da saúde dos trabalhadores do município de Santa Cruz foram discutidos, demonstrando e reforçando a necessidade que a questão saúde $\mathrm{x}$ trabalho seja entendida de forma ampla e integral, onde as diversas dimensões envolvidas na relação sejam incorporadas em qualquer ação que venha a interferir positivamente no processo de aprimoramento da produção industrial, sem com isso negligenciar o próprio homem e todas as suas peculiaridades.

Dessa forma, procuramos enfatizar que, embora haja uma busca por novas tecnologias que favoreçam o aprimoramento dos processos produtivos, a preocupação com a saúde dos funcionários deve ser considerada por todas as filosofias administrativas mais modernas, comprovando ser possível oferecer qualidade de vida aos que trabalham, e ao mesmo tempo gerar benefícios reais para a empresa.

\section{Referências}

1. Deliberato PCP. Fisioterapia Preventiva: Fundamentos e aplicações. São Paulo: Manole; 2002.

2. Antón AV et al. Prevention and Postural Education Programme in the Textile Sector. Fisioterapia 2002; 24: 63-9.

3. Salim CA. Doenças do Trabalho: exclusão, segregação e relações do gênero. São Paulo em Perspectiva 2003; 17: 11-24.
4. Merlo ARC. O trabalho entre prazer, sofrimento e adoecimento: a realidade dos portadores de lesões por esforços repetitivos. Psicologia \& Sociedade 2003; 15: 11736.

5. Cartaxo C. Estudo ergonômico do posto de trabalho do armador de laje - uma avaliação dos esforços físicos na coluna vertebral decorrentes de posturas de trabalho. [dissertação de mestrado] João Pessoa: Departamento de Engenharia de produção da Universidade Federal da Paraíba; 1997. 
6. Gómez-Conesa A. Factores posturales de riesgo para la salud. Fisioterapia 2002; 24: 23-32.

7. Corlett EN, Manenica I. The effects and measurement of working postures. Applied Ergonomics 1980; 11: 7-16.

8. Batista EB et al. Lesões por esforço repetitivo em digitadores de processamento de dados do Banesta. Londrina, Paraná, Brasil. Revista de Fisioterapia de São Paulo 1997; 4: 83-91.

9. Brisson $\mathrm{C}$ et al. Disability among female garment workers. A comparison with a national sample. Scand J Work Environ Health 1989; 15: 323-8.

10. Andersen JH, Gaarboe O. Prevalence of persistent neck and upper limb pain in a historical cohort of sewing machine operators. Am J Ind Med 1993; 24: 677-87.

11. Moraes MAA et al. Sintomas músculo-esqueléticos e condições de trabalho de costureiras de um hospital universitário. Rev Paulista de Enfermagem 2002; 21:24954.

12. Barreira THC. Um enfoque ergonômico para as posturas de trabalho. Rev Bras Saúde Ocupacional 1989; 17: 61-71.

13. Arnetz BB et al. Early workplace intervention for employees with musculoskeletal related absenteeism: a prospective controlled intervention study. Occup Environ Med 2003; 45: 499-506.

14. Szubert Z, Zyeinska Z. Analysis of work absenteeism due to illness in the provinces. Medicina Preventiva 1996; 47: $117-23$.

15. Gravina MER. LER - Lesões por esforços repetitivos: uma reflexão sobre os aspectos psicossociais. Saúde e Sociedade 2002; 11: 65-87.
16. Santos Filho SB, Barreto SM. Atividade ocupacional e prevalência de dor osteomuscular em cirurgiõesdentistas de Belo Horizonte, Minas Gerais, Brasil: Contribuição ao debate sobre os distúrbios osteomusculares relacionadas ao trabalho. Cad Saúde Pública 2001; 17: 181-93.

17. Westgaard RH, Jansen T. Individual and work related factors associated with symptoms of musculoskeletal complaints, II. Different risk factors among sewing machine operators. Br J Ind Med 1992: 49: 154-62.

18. Kaegaard A, Andersen JH. Musculoskeletal disorders of the neck and shoulders in female sewing machine operators: prevalence, incidence and prognosis. Occup Environ Med 2000; 57: 528-34.

19. Gamperiene M, Stigum H. Work related risk factors for musculoskeletal complaints in the spinning industry in Lithuania. Occup Environ Med 1999; 56: 411-6.

20. Kjaer M. From exercise physiology to sports medicine. Ugeskr Laeger 1999; 161: 6329-33.

21. Reis RJ et al. Perfil da demanda atendida em ambulatório de doenças profissionais e a presença de lesões por esforços repetitivos. Rev Saúde Pública 2000; 34: 292-8.

22. Orlando AR, King PM. Relationship of demographic variables on perception of fatigue and discomfort following prolonged standing under various flooring conditions. JOccup Rehab 2004; 14: 63-76.

23. Brulim C et al. Physical and psychosocial work-related risk factors associated with musculoskeletal symptoms among home care personnel. Scand Journal 1998; 12: 10410.

Recebido em: 23/09/05

Versão reformulada reapresentada em: 09/12/05

Aprovado em: 14/12/05 